\title{
Chromosome localization of microsatellite markers in the shrews of the Sorex araneus group
}

Patrick Basset $^{1 *}$, Glenn Yannic ${ }^{1}$, Fengtang Yang ${ }^{2,6}$, Patricia C. M. O’Brien ${ }^{2}$, Alexander S. Graphodatsky ${ }^{3}$, Malcolm A. Ferguson-Smith ${ }^{2}$, Gabriel Balmus ${ }^{2,5}$, Vitaly T. Volobouev ${ }^{4}$ \& Jacques Hausser ${ }^{1}$

${ }^{1}$ Department of Ecology and Evolution, Lausanne University, Biophore, 1015 Lausanne, Switzerland; Tel: +4121-6924186; Fax: +41-21-6924165; E-mail: Patrick.Basset@unil.ch; ${ }^{2}$ Centre for Veterinary Science, University of Cambridge, Cambridge, CB2 ITN, UK, ${ }^{3}$ Russian Academy of Science, Institute of Cytology and Genetics, Novosibirsk, 630090, Russia; ${ }^{4}$ Museum of Natural History, National Laboratory, Origin Struct \& Evolut Biodivers, 55 Rue Buffon, Paris, 75005, France; ${ }^{5}$ Faculty of Veterinary Medicine, Iasi, 700489, Romania; ${ }^{6}$ Wellcome Trust Sanger Institute, Wellcome Trust Genome Campus, Hinxton, Cambridge, CB10 1SA, UK

* Correspondence

Received 23 December 2005. Received in revised form and accepted for publication by Pat Heslop-Harrison 3 February 2006

Key words: flow-sorting, karyotype, microsatellite mapping, Sorex araneus

\begin{abstract}
The extremely high rate of karyotypic evolution that characterizes the shrews of the Sorex araneus group makes this group an exceptionally interesting model for population genetics and evolutionary studies. Here, we attempted to map 46 microsatellite markers at the chromosome arm level using flow-sorted chromosomes from three karyotypically different taxa of the Sorex araneus group ( $S$. granarius and the chromosome races Cordon and Novosibirsk of $S$. araneus). The most likely localizations were provided for 35 markers, among which 25 were each unambiguously mapped to a single locus on the corresponding chromosomes in the three taxa, covering the three sexual chromosomes (XY1Y2) and nine of the 18 autosomal arms of the $S$. araneus group. The results provide further evidence for a high degree of conservation in genome organization in the $S$. araneus group despite the presence of numerous Robertsonian rearrangements. These markers can therefore be used to compare the genetic structure among taxa of the $S$. araneus group at the chromosome level and to study the role of chromosomal rearrangements in the genetic diversification and speciation process of this group.
\end{abstract}

\section{Introduction}

Comparative gene mapping is of primary interest to understand the evolution of the mammalian karyotype (O’Brien et al. 1999). Among mammals, efforts have mostly been concentrated on humans or laboratory and farm animals, and have revealed surprising conservation in mammalian genome organizations. However, to get a satisfactory picture of the genome changes that have occurred during mammalian radiation, it is important to expand the range of orders examined (O'Brien et al. 2001). In this context the former Order Insectivora, defined by morphological characters, is of special interest. Morphologically, its members appear to be the closest to the ancestral eutherian condition (Nowak 1991), but DNA markers suggest their paraphyletic origin (Arnason \& Janke 2002, Murphy et al. 2004), placing the shrews, hedgehogs and moles in the order Eulipotyphla (i.e., core insectivores, Douady et al. 2002). 
Among the core insectivores the shrews of the Sorex araneus group have been subjected to detailed phylogenetic and population genetic analyses involving chromosome and genetic markers (for reviews see Searle \& Wójcik 1998). This monophyletic group consisting of 10 species is characterized by the particular sexual chromosome complex XY1Y2 in males (Sharman 1956) and by a spectacular karyotypic diversification, in spite of their high degree of morphological similarity (Zima et al. 1998). In the type species of this group, Sorex araneus, Robertsonian polymorphisms are particularly prevalent. The ancestral karyotype of this group is thought to consist of acrocentric chromosomes only. Repeated Robertsonian fusions led to the formation of various metacentric chromosomal complements seen in more than 60 different chromosomal races, each characterized by a particular set of metacentrics and acrocentrics (Wójcik et al. 2003). Apart from Robertsonian fusion, high-resolution chromosome analyses (Volobouev 1989, Volobouev \& Catzeflis 1989) suggested the occurrence of a few telomere-centromere translocations and centromeric shifts in some species. This outstanding karyotypic variation makes $S$. araneus an exceptionally interesting model species for population genetics and evolutionary studies.

Given its large distribution, its abundance, its ancestral mammalian morphology and its peculiar genome evolution, $S$. araneus represents an obvious 'type' species for the core insectivores. Consequently, a gene mapping project of the common shrew chromosomes was undertaken (Pack et al. 1995) and its genome selected as a candidate for complete sequencing (O'Brien et al. 2001, Pennisi 2004). The $S$. araneus genetic map currently contains 53 markers (Zhdanova et al. 2003) which mark the X (de) chromosome and seven out of the nine metacentric chromosomes of the Novosibirsk race. There are, however, some gaps to be filled in order to take full advantage of this map to address many important evolutionary problems. For example, a genetic map of microsatellite loci would provide a powerful tool to unravel the role of chromosomal rearrangements in the speciation process. Indeed, over the entire range of the $S$. araneus group, several populations characterized by different karyotypes come into contact and hybridize. Recently, new models of speciation have been developed to account for the proposed role of chromosomal rearrangements in speciation (Noor et al. 2001, Rieseberg 2001, Navarro \& Barton
2003); but in the case of the $S$. araneus group it is currently not possible to distinguish between a reproductive barrier caused by genetic effects or by karyotypic differences (Lugon-Moulin et al. 1999, Brünner et al. 2002). This question could be addressed with a battery of microsatellite markers mapped at the chromosome level. However, only four of these markers have been located so far (Zhdanova et al. 2003).

Traditionally, gene mapping in the $S$. araneus group dealt with somatic cell hybrid panels (Pack et al. 1995); but, due to numerous hidden chromosome rearrangements in panels, this method proved laborious (Zhdanova et al. 2003). Mapping accuracy would be further improved with the use of mostly acrocentric taxa, but only the all-metacentric $S$. araneus Novosibirsk chromosome race has been used so far.

In this study we attempted to map 46 microsatellite loci at the chromosome arm level using flowsorted chromosomes of three karyotypically different taxa of the $S$. araneus group. The genome-wide chromosomal correspondence among the three taxa studied here is well established (e.g., Volobouev 1989, Volobouev \& Catzeflis 1989). The first taxon is $S$. granarius, a species genetically very similar to S. araneus (Fumagalli et al. 1999), which is believed to have retained the ancestral karyotype of the S. araneus group (Wójcik \& Searle 1988, Volobouev 1989). Indeed, all its autosomes (except the smallest one $t u$ ) are in an acrocentric state. The second taxon, $S$. araneus Cordon, is among one of the most acrocentric $S$. araneus chromosome races since most of its autosomes (except for the two large $a f$ and $b c$, the smallest one $t u$ and the polymorphic $j / l$ ) are acrocentric. The third taxon was the $S$. araneus Novosibirsk race, the traditional 'gene mapping race' for the $S$. araneus group, with all its autosomes being metacentric.

\section{Material and methods}

\section{Chromosome isolation}

Chromosomes from fibroblast cell lines of one female of $S$. granarius and S. araneus Cordon and one male of $S$. araneus Novosibirsk were sorted on a bivariate fluorescence-activated flow sorter as described previously (Yang et al. 1995). Their respective karyo- 
types consisted of $2 \mathrm{n}=36(a, b, c, f, g, h, i, j, k, l, m$, $n, o, p, q, r, t u, \mathrm{X}(d e)), 2 \mathrm{n}=30(a f, b c, g, h, i, j l, k, m$, $n, o, p, q, r, t u, \mathrm{X}(d e)$; Figure $1 \mathrm{~A})$ and $2 \mathrm{n}=21$ (af, $b c, g o, h n, i k, j l, m p, q r, t u, \mathrm{X}(d e), \mathrm{Y} 1(s), \mathrm{Y} 2(d))$ chromosomes. Note that each of the 21 chromosome arms of the $S$. araneus group is labeled by a letter $(a-u)$, with ' $a$ ' being the largest arm and ' $u$ ' the smallest (Searle et al. 1991) and that the corresponding chromosomes among the three taxa were each named using the same letter. Chromosomespecific DNA from these individuals was obtained by degenerate oligonucleotide-primed PCR (DOP-PCR) amplification of flow-sorted chromosomes following standard procedures (Telenius et al. 1992, Yang et al. 1995). The characterization and purity of each chromosome sort was checked by fluorescence in-situ hybridization (FISH). Painting probes were hybridized to standard metaphase preparations of their respective species and assigned to specific chromosomes by digital imaging according to the published protocols (Yang et al. 1999).

\section{Sorex araneus group microsatellite loci}

Twenty-two markers have already been described in the S. araneus group (Wyttenbach et al. 1997,
Balloux et al. 1998, 2000, Lugon-Moulin et al. 2000, Basset et al. 2006). Mapping efficiency is sometimes low (Zhdanova et al. 2003); therefore this number was not sufficient to ensure that our microsatellite genetic map would mark most of the chromosomes. For this reason we described another 24 loci extracted from four microsatellite-enriched libraries developed by a commercial company (Genetic Identification Services, Inc., Chatsworth, CA). These loci were found to be highly polymorphic and add to the 22 previously characterized loci to give a total of 46 microsatellite markers described for the $S$. araneus group (Table 1).

\section{Mapping microsatellite loci to chromosome}

Flow-sorted chromosomes were amplified a second time by DOP-PCR (Telenius et al. 1992). This second DOP-PCR amplification step was necessary to have enough material and sensitivity to test all loci. Each chromosome-specific DNA was then screened for the presence of each $S$. araneus group microsatellite locus by standard PCR using conditions as described in Table 1.

PCR conditions of formerly described markers are given in Wyttenbach et al. (1997), Balloux et al.
A

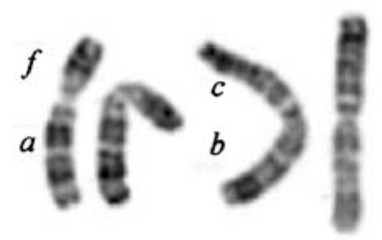

$g(2)$

a

$m$
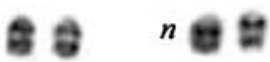

$q$

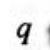

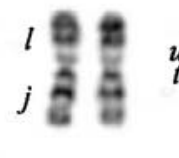
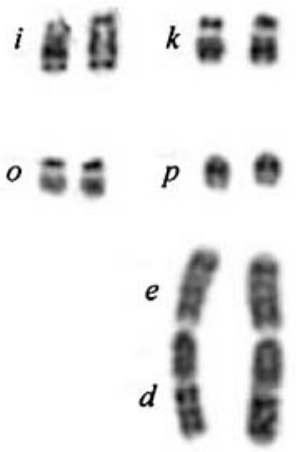

B

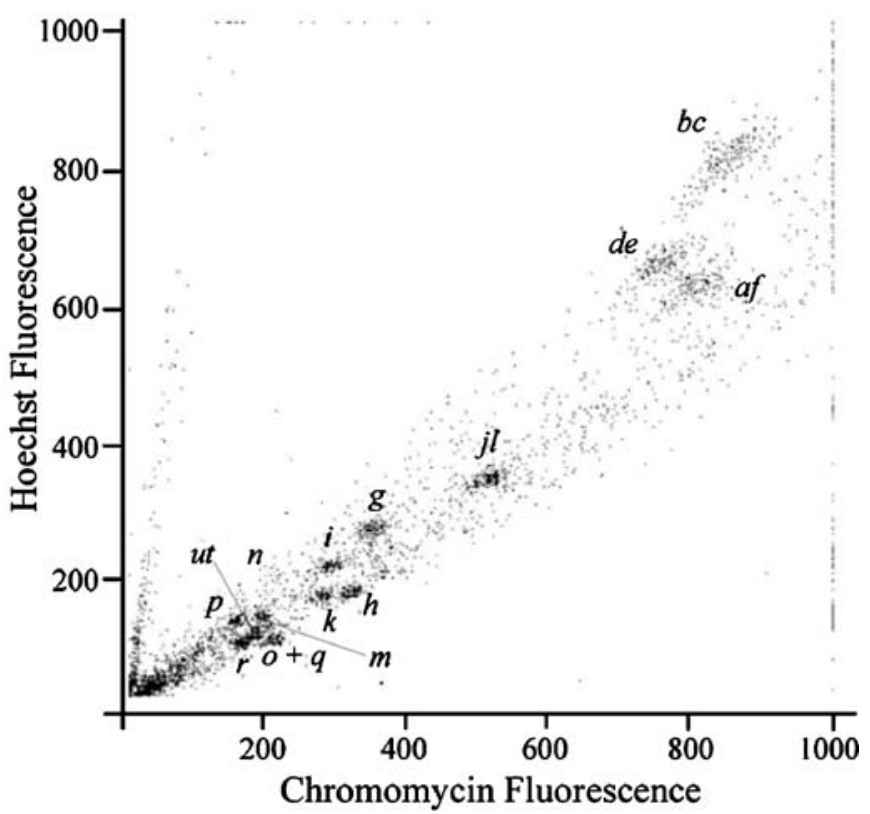

Figure 1. G-banded karyotype (A) and flow karyotype (B) of the female Sorex araneus Cordon. 
Table 1. PCR primers and amplification conditions used for mapping the Sorex araneus group microsatellite loci on Sorex chromosomes

\begin{tabular}{|c|c|c|c|c|c|c|}
\hline MS & Accession number & Primers $\left(5^{\prime}-3^{\prime} ;\right.$ for/rev $)$ & Motifs $^{\mathrm{a}}$ & $\begin{array}{l}\text { Annealing } \\
\left({ }^{\circ} \mathrm{C}\right)\end{array}$ & $\begin{array}{c}\mathrm{MgCl}_{2} \\
(\mathrm{mM})\end{array}$ & Refs \\
\hline $\mathrm{L} 2$ & U82710 & $\begin{array}{l}\text { CAAAAACAAAAAAGAAGAAAGAAG } \\
\text { TCTTTATCСТCCATTCССТC }\end{array}$ & $(\mathrm{GGA}) 12$ & 55 & 1.5 & 1 \\
\hline L9 & U82711 & $\begin{array}{c}\text { TCATGGACTTTTCTGTGCTG } \\
\text { CTTTGGCATGAATTTGCC }\end{array}$ & (AC)29 & 55 & 1.0 & 1 \\
\hline L13 & AF175741 & $\begin{array}{c}\text { TATAACTGTTATCTCACAGCGATTCA } \\
\text { ATCCATCTTTATCTTTTCCATTGC }\end{array}$ & $(\mathrm{AATT}) 6$ & 55 & 1.5 & 3 \\
\hline L14 & AF032911 & $\begin{array}{l}\text { AGGGAGGGAAACTTGTTAAAGG } \\
\text { GTGAGGTCCTGGAATAGTGTCC }\end{array}$ & $(\mathrm{AC}) 14$ & 55 & 1.0 & 2 \\
\hline L16 & U82712 & $\begin{array}{l}\text { TCAGAGTCAGAATTTCTAATTTGGC } \\
\text { TTAGTGTATTATGACAGATGCGGG }\end{array}$ & (AC) 16 & 55 & 1.5 & 1 \\
\hline L33 & AF032912 & $\begin{array}{l}\text { TGATTGTATGGAAAAAAGCCG } \\
\text { ATACTAAGACCACCCTACCAATGC }\end{array}$ & (AC) 19 & 55 & 1.5 & 2 \\
\hline L45 & U82713 & $\begin{array}{l}\text { CTTAAACGTTCTTATCTATTTGGTTG } \\
\text { GACATATGTGCACTATGAAATTATTG }\end{array}$ & $(\mathrm{AC}) 10$ & 55 & 1.5 & 1 \\
\hline L57 & U82714 & $\begin{array}{l}\text { CTGTTTTTCTGTCCCTCATAGC } \\
\text { TGTCCTAGTGACATTATCCTATTGG }\end{array}$ & $(\mathrm{AC}) 10$ & 55 & 2.0 & 1 \\
\hline L62 & U82715 & $\begin{array}{l}\text { CAGTCTCTCACTGTGGCACTATG } \\
\text { GTCATTCTGGATAAGAACCATATGC }\end{array}$ & (AC) 16 & 55 & 1.5 & 1 \\
\hline L67 & U82716 & $\begin{array}{l}\text { GAAGTGATACATGAGTGCATGAG } \\
\text { GTTGTTAACAAGAGAGGTATTACACC }\end{array}$ & $(\mathrm{AC}) 17$ & 55 & 1.5 & 1 \\
\hline L68 & AF032913 & $\begin{array}{l}\text { TCATGGTCATTTCATCACATACC } \\
\text { GTAGATGTTGCCACTGGTGG }\end{array}$ & $(\mathrm{AC}) 14$ & 55 & 1.5 & 2 \\
\hline L69 & U82717 & $\begin{array}{l}\text { CTTTATGGTAGAAAATGGTG } \\
\text { GACCATATACTAAGTTGTTTTG }\end{array}$ & $(\mathrm{AC}) 17$ & 57 & 1.5 & 1 \\
\hline L92 & AF032914 & $\begin{array}{l}\text { ACTGGTGCCCAATCGATAAG } \\
\text { GAGAATTGTTGGATGTGCCC }\end{array}$ & $(\mathrm{AC}) 7$ & 55 & 1.5 & 2 \\
\hline L97 & AF032915 & $\begin{array}{l}\text { ATTCTCGTGGGTAGACCGTG } \\
\text { ATAAATGTGGGAAATGGACAGG }\end{array}$ & $(\mathrm{AC}) 56$ & 55 & 1.5 & 2 \\
\hline L99 & AF175744 & $\begin{array}{l}\text { AAATAATTTCTTCCTGGCAAG } \\
\text { ATAAATGCAGCAAAGTTATAAACTT }\end{array}$ & $(\mathrm{AC}) 6$ & 55 & 1.5 & 3 \\
\hline L8Y & AF175743 & $\begin{array}{l}\text { ССTCTTTTGTTTCTCATCATTTTC } \\
\text { AATGATGAAGTGGATGAGTTAGATACC }\end{array}$ & $(\mathrm{GAA}) 20$ & 55 & 1.5 & 4 \\
\hline $\mathrm{A} 8$ & DQ247975 & $\begin{array}{l}\text { CTACGCGCCTTCTTTCAGTC } \\
\text { GAAGCTGTCCACTGTGTAAACG }\end{array}$ & (CA)26 & 57 & 1.5 & 6 \\
\hline A25 & DQ247976 & $\begin{array}{l}\text { GGCAGTGCTCAGGGATAAC } \\
\text { AGTGAGGACAGAATTTCAGGTG }\end{array}$ & (CA) 25 & 57 & 1.0 & 6 \\
\hline B3 & DQ074646 & $\begin{array}{l}\text { CTTGCCACATTCCCACATC } \\
\text { AGCCCCACAGCTTTCTCC }\end{array}$ & (GA)30 & 57 & 1.0 & 5 \\
\hline B5 & DQ074647 & $\begin{array}{l}\text { ATGTCTTGCTGGCTGAAGG } \\
\text { CTGCTGTTCACAAACTCCAAG }\end{array}$ & (CA)12(GA) 19 & 55 & 1.5 & 5 \\
\hline B7 & DQ247977 & $\begin{array}{l}\text { AGACGCCCTTGTTCTCTCC } \\
\text { CCCAGGACTTTCGGTTCTTAC }\end{array}$ & (GA)29 & 60 & 1.5 & 6 \\
\hline B10 & DQ074648 & $\begin{array}{l}\text { CTCCAAACCCTAACACTCTGTC } \\
\text { TTCACGTGTTCTTTGCTTCC }\end{array}$ & (GA)30 & 55 & 1.5 & 5 \\
\hline B12 & DQ247978 & $\begin{array}{l}\text { GGGGCTTTCTCCACTCTTG } \\
\text { TGCTCAGACCTTGATTAGACACTC }\end{array}$ & (GA)34 & 60 & 1.5 & 6 \\
\hline B15 & DQ074650 & $\begin{array}{l}\text { GTAGAGTTGCTGGCTCAAAGG } \\
\text { ATGGGAAGACATTGGATTGG }\end{array}$ & (GA)31 & 55 & 1.5 & 5 \\
\hline B30 & DQ247979 & $\begin{array}{l}\text { TCTCCCTTATCCCGCTGTC } \\
\text { ACGAAAGGCTGCAACTCAAC }\end{array}$ & (GA)26 & 55 & 1.5 & 6 \\
\hline C5 & DQ074649 & $\begin{array}{l}\text { TAGATGACTCTGTGTTCAGGC } \\
\text { GTTGGGAAGGTAAGATCAGG }\end{array}$ & $(\mathrm{GA}) 35(\mathrm{CA}) 12$ & 55 & 1.5 & 5 \\
\hline C19 & DQ074651 & $\begin{array}{l}\text { TGCCATAAACACCACTTACC } \\
\text { GTGATCAATACCCTGTGGAG }\end{array}$ & (GA) 22 & 60 & 1.5 & 5 \\
\hline
\end{tabular}


Table 1. (continued)

\begin{tabular}{|c|c|c|c|c|c|c|}
\hline MS & Accession number & Primers $\left(5^{\prime}-3^{\prime} ;\right.$ for/rev $)$ & Motifs $^{\mathrm{a}}$ & $\begin{array}{c}\text { Annealing } \\
\left({ }^{\circ} \mathrm{C}\right)\end{array}$ & $\begin{array}{c}\mathrm{MgCl}_{2} \\
(\mathrm{mM})\end{array}$ & Refs \\
\hline $\mathrm{C} 25$ & DQ247980 & $\begin{array}{l}\text { CCCAGGCATAAGTTTCAGG } \\
\text { TGTGAACTGTGGTGGATAGATG }\end{array}$ & $(\mathrm{GA}) 29$ & 57 & 1.5 & 6 \\
\hline $\mathrm{C} 100$ & DQ247981 & $\begin{array}{l}\text { CTCGGTGTTTCTACGAT } \\
\text { CAGAGATAGAAGAGGCCAAG }\end{array}$ & (GA)21 & 55 & 2.0 & 6 \\
\hline $\mathrm{C} 117$ & DQ247982 & $\begin{array}{l}\text { TAGATGACCAGGATGGAG } \\
\text { ACAGAGCTGGGAATCAGT }\end{array}$ & $(\mathrm{GAT}) 24$ & 55 & 1.5 & 6 \\
\hline C119 & DQ247983 & $\begin{array}{l}\text { CCAGCCTTTACTTCTGCTAC } \\
\text { TGGGTCTCATTCCTCTGAC }\end{array}$ & $(\mathrm{CAT}) 29$ & 50 & 1.5 & 6 \\
\hline $\mathrm{C} 122$ & DQ247984 & $\begin{array}{l}\text { AGTTTTCTTCTCGCCCGTCT } \\
\text { CCACTGTGCCAAGGATAGTT }\end{array}$ & (CT) 17 & 57 & 1.5 & 6 \\
\hline $\mathrm{C} 151$ & DQ247985 & $\begin{array}{r}\text { CAACGGAGACATTACTGGTG } \\
\text { CCAAACTCAAAGGCAGGA }\end{array}$ & (TGA)30 & 55 & 1.5 & 6 \\
\hline $\mathrm{C} 171$ & DQ247986 & $\begin{array}{c}\text { GTGACTGTTCCCATGATGAC } \\
\text { ACCAATGTCCCCAGTTTC }\end{array}$ & (GA)25 & 55 & 1.5 & 6 \\
\hline $\mathrm{C} 240$ & DQ247987 & $\begin{array}{l}\text { GGGTTCAATCTCCAACATCC } \\
\text { ATCCTGCCСТTCTTTTCCTC }\end{array}$ & $(\mathrm{GA}) 22$ & 55 & 1.5 & 6 \\
\hline D11 & DQ247988 & $\begin{array}{l}\text { GTGTCGAGAGTCGGAAAACC } \\
\text { AGCCAGGAACAAGCCCTAC }\end{array}$ & (TAGA) 15 & 57 & 1.5 & 6 \\
\hline $\mathrm{D} 23$ & DQ247989 & $\begin{array}{l}\text { ATGGTGGAAAGGCTCAAG } \\
\text { AAAAGCAGTATTGGGTCTGG }\end{array}$ & (TAGA) 23 & 57 & 1.5 & 6 \\
\hline D24 & DQ247990 & $\begin{array}{c}\text { CCCAGAGTTACCTTTGAGATATGC } \\
\text { TCAATTTTCCCTGGAGGATG }\end{array}$ & (GA)38 & 55 & 1.5 & 6 \\
\hline D29 & DQ247991 & $\begin{array}{l}\text { GGAAGCAGCGTGAGACTACC } \\
\text { AATGGAGACGTGACTGAGACC }\end{array}$ & $(\mathrm{ATC}) 9$ & 65 & 1.5 & 6 \\
\hline D103 & DQ247992 & $\begin{array}{l}\text { TTATGCCACTGATACACCAA } \\
\text { ATCCAAAAGGGTTTCCTTAC }\end{array}$ & $(\mathrm{CTAT}) 12$ & 55 & 2.0 & 6 \\
\hline D106 & DQ247993 & $\begin{array}{l}\text { ATTTCTCCCTTCAATCTGGT } \\
\text { AGGAGTACCTCTGGGTGTG }\end{array}$ & $(\mathrm{CTAT}) 10$ & 55 & 2.0 & 6 \\
\hline D107 & DQ247994 & $\begin{array}{l}\text { AGGAAGACTGGGGGTATGTT } \\
\text { TAGGTCTGCTGCCTGCAT }\end{array}$ & $(\mathrm{CTAT}) 17$ & 55 & 2.0 & 6 \\
\hline D109 & DQ247995 & $\begin{array}{l}\text { TGAACTTGGGAGATGCAAT } \\
\text { ATAGGAGAGGGCAAGCAG }\end{array}$ & $\begin{array}{l}(\mathrm{CTAT}) 15 \mathrm{CG} \\
\quad(\mathrm{CT}) 15\end{array}$ & 55 & 2.0 & 6 \\
\hline D110 & DQ247996 & $\begin{array}{l}\text { TGTTTTGGTTGAGGTTGG } \\
\text { TCACACGCCATCAGTAAGT }\end{array}$ & $(\mathrm{CTAT}) 36$ & 55 & 2.0 & 6 \\
\hline D112 & DQ247997 & $\begin{array}{l}\text { GCAAACTACCTGTGGCGTATT } \\
\text { CCAGCCCTCTTATGAAACTCTT }\end{array}$ & $(\mathrm{CTAT}) 20$ & 60 & 2.0 & 6 \\
\hline D138 & DQ247998 & $\begin{array}{l}\text { ACCTGGAGTGACAGTGAGC } \\
\text { GGGTGCTGGAGTGACAGTAT }\end{array}$ & $(\mathrm{CTAT}) 21$ & 55 & 1.5 & 6 \\
\hline
\end{tabular}

References: (1) Wyttenbach et al. 1997, (2) Balloux et al. 1998, (3) Lugon-Moulin et al. 2000, (4) Balloux et al. 2000, (5) Basset et al. 2006; (6) This study.

${ }^{\mathrm{a}}$ Represents the size of the original clone.

(1998, 2000), Lugon-Moulin et al. (2000) and Basset et al. (2006).

PCR conditions of the markers described in this study were as follows: $0.2 \mathrm{mM}$ dNTPs, $0.325 \mu \mathrm{M}$ of each primer, $1 \times$ PCR buffer (Qiagen) and $0.5 \mathrm{U}$ Taq polymerase (Qiagen). $\mathrm{MgCl}_{2}$ concentration as well as annealing temperature varied (Table 1). For all primers, PCR amplifications were performed on 20-50 ng of DOP-PCR product in a final volume of $20 \mu \mathrm{l}$, and cycling was carried out in a PE 9700 thermal cycler (Applied Biosystems) using the cycling profile: $95^{\circ} \mathrm{C}$ for $5 \mathrm{~min} ; 35$ cycles of $30 \mathrm{~s}$ at $94^{\circ} \mathrm{C}, 30 \mathrm{~s}$ at the annealing temperature, $30 \mathrm{~s}$ at $72^{\circ} \mathrm{C}$; and a final extension at $72^{\circ} \mathrm{C}$ for $4 \mathrm{~min}$.

To guarantee correct assignments, each locus was tested against each chromosome sort of the three taxa studied, two to four times. Preliminary analyses showed that unspecific amplifications were sometimes observed in several chromosome sorts. Therefore, we used whole DNA from sorted individuals as 
positive controls whose amplification product sizes were used as references. To better estimate the size of the amplification product, one primer of each pair was labeled with a fluorescent dye on the $5^{\prime}$ end, and run on an ABI 377XL automated sequencer (Applied Biosystems). Data collection, sizing and analyses of PCR products were done using GENESCAN software (Applied Biosystems).

\section{Results}

\section{Chromosome flow-sorting and characterization} of flow karyotype

Bivariate chromosome flow-sorting allowed chromosome-specific sorts for the three taxa to be obtained. Sixteen distinct peaks were identified in the flow karyotype of $S$. granarius, 14 in $S$. araneus Cordon (Figure 1B) and 12 in S. araneus Novosibirk (data not shown). To assign the content of each peak to particular chromosome(s), painting probes from each peak were hybridized to metaphase preparations of each taxon (see Figure 2 for examples in a male of S. araneus Cordon). In S. granarius, 12 peaks each contained a single type of chromosome, whereas the other four peaks each contained multiple types of chromosomes (i.e., $j+k+l, r+t u, q+r, m+o$ ). In addition, chromosomes $m$ and $o$ were each represented in a second individual peak. In $S$. araneus Cordon, a single type of chromosome was found in 13 peaks and one peak contained two types of chromosomes $(o+q)$. Finally, all 12 peaks of $S$. araneus Novosibirsk each contained a single type of chromosome. Most chromosomes could reliably be assigned to a specific flow peak; therefore, these flow-sorted chromosomes can be used for genetic mapping purposes.

\section{Microsatellite mapping}

The mapping results of the 46 microsatellite loci for the three Sorex taxa are summarized in Table 2. Among the 41 loci showing positive amplification in $S$. granarius, 26 were each assigned to only one chromosome. For the S. araneus Cordon and Novosibirsk, 38 and 40 loci showed positive amplification, respectively, and 23 and 21 loci were each assigned to a single chromosome respectively. These cases clearly provide the best evidence of presence of the locus on a particular chromosome, particularly when different taxa give the same assignment on their corresponding chromosomes. Such cases where a locus was assigned to multiple chromosomes could be assigned with less confidence.

The most likely localizations have been deduced for 35 microsatellite markers, 25 of which each showed a similar single assignment in different taxa (bold assignments, Table 2) and are therefore considered unambiguously mapped. At least nine of the 18 autosomal chromosome arms found in the $S$. araneus group were represented by these markers: $a$ (3 loci), $b$ (5), $c$ (1), $f(3), g(1), \mathrm{h}(1), j$ or $l(2), n$ (1) and $o(2)$. We were not able to discriminate between the chromosome arms of the metacentric chromosome $j l$ since these arms were part of the same chromosome sort in S. granarius. The remaining six loci mapped to the sex chromosomes. The chromosome $\mathrm{X}(d e)$ is the product of a Robertsonian fusion between the 'original' mammalian $\mathrm{X}$ (most of arm $e$ ) and an autosome (all of arm $d$ and part of arm $e$; Pack et al. 1993). Chromosome $\mathrm{Y} 2$ in males corresponds to this autosome and is therefore homologous to chromosome arm $d$. Four loci (L57, C19, C171 and D138) were assigned to chromosomes $\mathrm{X}$ and $\mathrm{Y} 2$ in $S$. araneus Novosibirsk and probably map to the chromosome arm $d$. One locus (L13) was assigned only to the $\mathrm{X}$ chromosome in the same species, suggesting localization on chromosome arm $e$, the 'true' X chromosome. Finally, as expected, the malespecific locus L8Y (Balloux et al. 2000) mapped to chromosome Y1, the true Y. The distribution of our mapped loci was not significantly different from the expected null distribution according to chromosome arm size $\left(\chi^{2}=0.584\right.$, d.f. $\left.=17 ; p=\mathrm{NS}\right)$. Microsatellite loci thus did not appear to cluster on any particular chromosome. Only one locus assigned to single chromosome sorts in the three taxa showed discordance among the taxa (locus D11 mapped on chromosome $b$ in $S$. granarius and af in $S$. araneus Cordon and Novosibirsk).

\section{Discussion}

Data concerning genetic exchange between chromosome arms in the $S$. araneus group are scarce. Using high-resolution chromosome analysis, Volobouev \& Catzeflis (1989) suggested only a few centromeric shifts between $S$. granarius and $S$. araneus. Our study 

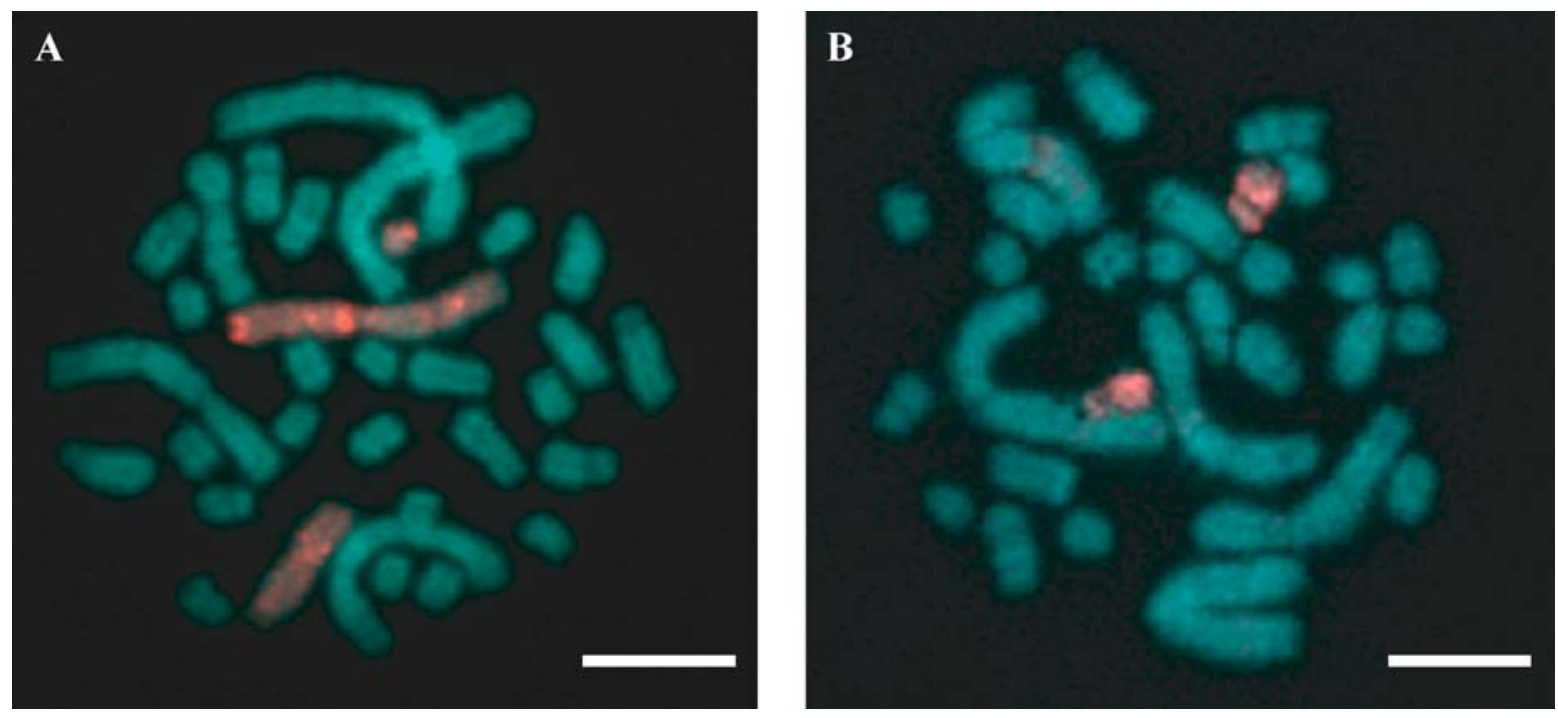

Figure 2. Examples of Sorex araneus Cordon male metaphase spreads showing fluorescence of individual chromosome painted by chromosome-specific probes generated from flow-sorted chromosomes: (A) Chr X (de); (B) Chr $k$. (Scale bars represent $10 \mu \mathrm{m}$.)

corroborates these findings and suggests that, despite the presence of numerous Robertsonian rearrangements, the organization of the genome in the S. araneus group is well conserved. Consequently, this high level of conservation suggests that our markers can be used to study and compare the genetic structure within and among the different species and chromosome races of the $S$. araneus group at the level of individual chromosomes.

Due to PCR amplifications in more than one chromosome-specific sort, several loci could not be each unambiguously assigned to a specific chromosome (e.g., question marks in Table 2). Interestingly, these loci were in general ambiguous in all three taxa. Several non-exclusive reasons may explain this pattern: i.e., lack of specificity of markers, contamination between sorts or genomic rearrangements.

First, the competition among binding sites on a single chromosome is probably weaker than over the whole genome. Therefore, the specificity of a primer pair may decrease when tested on isolated chromosomes and allow unspecific amplification. Nevertheless, most of our attempts to increase PCR specificity and design new primer pairs did not improve our capacity to assign loci to a single chromosome sort.

Second, the purity by which individual chromosomes can be sorted can approach but never reach 100\% (Ferguson-Smith 1997, Doležel et al. 2004;
Ibrahim \& van den Engh 2004). Therefore, chromosomes of similar sizes and GC/AT ratios could contaminate the desired chromosome sort. However, some markers were mapped to chromosomes that are quite distant on the flow karyotype. In such cases the flow rate might have been too fast, allowing more than one chromosome to be sorted together. Alternatively, the fragmentation and/or clumping of chromosomes might have altered their size and caused them to be sorted in the wrong sort. No apparent contaminations (except sorting of multiple chromosomes in several sorts) were detected by our painting experiments; but these experiments may fail to detect low-level DNA contamination, which is not the case in PCR.

Finally, sequence duplication is common in the mammalian genome (Samonte \& Eichler 2002; Thomas et al. 2004). Although the evolution of duplicated non-coding regions (including microsatellites) is still poorly investigated, it is likely that some of our loci belong to such regions. When a locus with high mutation rate (such as microsatellites) is duplicated, it is expected that some individuals bear more than two alleles. The locus C122 probably illustrates this situation since several tri- or tetraploid individuals were detected for this locus. Additionally, this locus was assigned (after numerous attempts to increase PCR specificity) to the same two chromosome arms ( $a$ and $g$ ) in the three taxa. Contamination 
Table 2. Assignment results of the 46 microsatellite markers in the three karyotypic taxa Sorex granarius, S. araneus Cordon and S. araneus Novosibirsk

\begin{tabular}{|c|c|c|c|c|}
\hline Locus & Sorex granarius & S. araneus Cordon & S. araneus Novosibirsk & Most likely localization \\
\hline L16 & $a$ & $a f$ & af & $a$ \\
\hline D107 & $a$ & af & af & $a$ \\
\hline D112 & $a$ & $a f$ & $a f$ & $a$ \\
\hline L2 & $b$ & n.a. & $b c$ & $b$ \\
\hline L68 & $b$ & $b c$ & $b c$ & $b$ \\
\hline $\mathrm{B} 10$ & $b$ & $b c$ & $b c$ & $b$ \\
\hline C100 & $b$ & $b c$ & $b c$ & $b$ \\
\hline C117 & $b$ & $b c$ & $b c$ & $b$ \\
\hline L45 & $b / i / d e$ & $b c / a f / j l / d e / u t$ & $b c / a f$ & $b$ \\
\hline L92 & $b / d e$ & $b c / d e$ & $a f / b c$ & $b$ \\
\hline L97 & - & $b c / i$ & $b c$ & $b$ \\
\hline D110 & $b / d e / c$ & $b c / j l / u t$ & $b c / a f / q r / d e / d$ & $b$ \\
\hline L9 & $c$ & $b c$ & - & $c$ \\
\hline L57 & $d e$ & $d e$ & de/d & $d$ \\
\hline C19 & $d e$ & $d e$ & deld & $d$ \\
\hline $\mathrm{C} 171$ & $d e$ & n.a. & de/d & $d$ \\
\hline D138 & $d e$ & $d e$ & de/d & $d$ \\
\hline L13 & $d e$ & $d e$ & $d e$ & $e$ \\
\hline L69 & $f$ & - & $a f$ & $f$ \\
\hline B3 & $f$ & $a f$ & af & $f$ \\
\hline B 15 & $f$ & af & - & $f$ \\
\hline C119 & $b / f$ & n.a. & af/ik/go & $f$ \\
\hline A25 & $b / f$ & $a f / r / m p$ & $a f / m p$ & $f$ \\
\hline D23 & $f / j, k, l$ & - & af/go & $f$ \\
\hline L62 & $g$ & $g$ & go & $g$ \\
\hline B7 & n.a. & $g / k$ & af/go & $g$ \\
\hline D106 & $h$ & $h$ & - & $h$ \\
\hline A8 & - & $j l$ & - & $j l$ \\
\hline D24 & $j, k, l$ & jl & $j l$ & $j l$ \\
\hline L33 & $a / m, o$ & $i / o, q / m$ & $m p$ & $m$ \\
\hline L99 & $n$ & $n$ & $h n$ & $n$ \\
\hline B30 & $o$ & $o, q$ & go & $o$ \\
\hline D109 & $f$ & $o, q$ & go & $o$ \\
\hline L14 & flm/o/de & $g / i / o, q$ & af/go & $o$ \\
\hline L8Y & n.a. & n.a. & $s$ & $s$ \\
\hline D11 & $b$ & $a f$ & $a f$ & $?$ \\
\hline $\mathrm{C} 5$ & a/i/de & - & - & $?$ \\
\hline $\mathrm{C} 25$ & $f / i$ & $a f / i$ & af/mp/ik/go/yl & $?$ \\
\hline $\mathrm{C} 122$ & $a / g$ & af $/ g$ & af/go & $?$ \\
\hline L67 & $b / i / q, r$ & $a f / g$ & $b c / q r$ & $?$ \\
\hline B5 & $m / i$ & $a f / j l / g / p$ & $a f / m p / y 2$ & $?$ \\
\hline B12 & n.a. & $\mathrm{r} / \mathrm{m}$ & n.a. & $?$ \\
\hline $\mathrm{C} 240$ & $i$ & $j l / p$ & af & $?$ \\
\hline C151 & $a / f$ & n.a. & $a f / m p$ & $?$ \\
\hline D29 & $b / d e / m, o$ & $u t$ & $a f / m p$ & $?$ \\
\hline D103 & $i$ & $m / u t$ & $m p / a f$ & $?$ \\
\hline
\end{tabular}

Autosomal and sexual chromosome arm names follow the traditional chromosome nomenclature in the S. araneus group (Searle et al. 1991). Unsorted chromosomes and multiple assignments are indicated by commas and slashes respectively. No amplification of correct size on sorted chromosome (-) nor on both sorted chromosome and positive control (n.a.). Most likely chromosome or chromosome arm assignments are indicated in the last column of the table: in bold, loci showing corresponding single assignment in different taxa; question marks, loci which cannot be assigned with confidence. 
between these chromosomes in the three taxa is highly unlikely since these chromosome arms are combined in acrocentric or metacentric chromosomes of different size (i.e., $a / g$ in $S$. granarius, af $/ g$ in $S$. araneus Cordon and af/go in $S$. araneus Novosibirsk).

As previously mentioned, four microsatellite loci have already been mapped by Zhdanova et al. (2003) using somatic cell hybrid panels of $S$. araneus Novosibirsk. For two loci the situation was consistent between the two studies: locus L16 was unambiguously mapped to chromosome af and the most likely localization for locus L14 is on chromosome arm $o$. The two other loci (L92 and L67), however, give conflicting results. These loci were respectively mapped to chromosome af and $j l$ by Zhdanova $e t$ al. (2003) whilst both loci amplified in more than one chromosome sort in our study. Our results for locus L92 point towards chromosome arm $b$, although an amplification product was also detected on chromosome af in S. araneus Novosibirsk. As for locus L67, the situation is even more complex: our results do not allow for any conclusions but no amplification on chromosome $j l$ in any of the three taxa was detected. A possible explanation for these discrepancies could be the high rate of hidden chromosomal rearrangements noticed in the shrew somatic cell hybrid panels (Zhdanova et al. 2003). However, as already mentioned, our method suffers from several uncertainties that cannot be ruled out for these loci.

\section{Conclusions}

PCR-based localization of genetic markers on flowsorted chromosomes has been used in a variety of groups (e.g., Sargan et al. 2000, Doležel et al. 2004). Although this method suffers from several limitations (e.g., genetic markers are assigned to chromosomes but not positioned on them), we have been able to assign unambiguously 25 microsatellite markers on three karyotypically different taxa of the $S$. araneus group. Also, the use of two particularly acrocentric taxa allowed for most assignments at the chromosome arm level. This last point should not be underestimated since this group shows an extraordinarily large number of Robertsonian metacentrics. Therefore we provide a microsatellite markers map that includes the Y chromosome (Y1), the two arms $d$ and $e$ of the $\mathrm{X}$ chromosome and at least nine of the 18 autosomal chromosome arms observed in this group.

The role of chromosomal rearrangements in the speciation process is still subject to controversy (for recent review see Coyne \& Orr 2004). Recently, several studies used mapped genetic markers to show that some genomic regions experience stronger barriers to gene flow than others and that these regions are linked to rearranged chromosomes (e.g., Rieseberg et al. 1999, Panithanarak et al. 2004). As previously mentioned, the outstanding karyotypic variation of the $S$. araneus group makes it an interesting model for studying the role of chromosomal rearrangement in the speciation process. Our results show a high level of conservation in the localization of markers among the studied taxa. They will therefore provide useful tools in both comparison of genetic structure among taxa at the individual chromosome level, and evaluation of the role of chromosomal rearrangements in the genetic diversification and speciation process of this group.

\section{Acknowledgements}

We are particularly grateful to Aline Dépraz, Jeremy Searle and an anonymous reviewer for helpful comments on the manuscript. This work was supported by the Swiss NSF (grants no: 3100-05943), a Société Académique Vaudoise (Switzerland) grant to P.B. and a Fondation Agassiz (Switzerland) grant to P.B.

\section{References}

Arnason U, Janke A (2002) Mitogenomics analyses of eutherian relationships. Cytogenet Genome Res 96: 20-32.

Balloux F, Ecoffey E, Fumagalli L, Goudet J, Wyttenbach A, Hausser J (1998) Microsatellite conservation, polymorphism, and GC content in shrews of the genus Sorex (Insectivora, Mammalia). Mol Biol Evol 15: 473-475.

Balloux F, Brünner H, Lugon-Moulin N, Hausser J, Goudet J (2000) Microcatellite can be misleading: an empirical and simulation study. Evolution 54: 1414-1422.

Basset P, Yannic G, Hausser J (2006) Genetic and karyotypic structure in the shrews of the Sorex araneus group: Are they independent? Mol Ecol (In press). 
Brünner H, Lugon-Moulin N, Hausser J (2002) Alps, genes, and chromosomes: their role in the formation of species in the Sorex araneus group (Mammalia, Insectivora), as inferred from two hybrid zones. Cytogenet Genome Res 96: 85-96.

Coyne JA, Orr AH (2004) Speciation. Sunderland, Mass.: Sinauer Associates.

Doležel J, Kubalákova M, Bartoš J, Macas J (2004) Flow cytogenetics and plant genome mapping. Chromosom Res 12 : 77-91.

Douady CJ, Chatelier PI, Madsen O et al. (2002) Molecular phylogenetic evidence confirming the Eulipotyphla concept and in support of hedgehogs as the sister group to shrews. Mol Phylogenet Evol 25: 200-209.

Ferguson-Smith MA (1997) Genetic analysis by chromosome sorting and painting: phylogenetic and diagnostic applications. Eur J Hum Genet 5: 253-265.

Fumagalli L, Taberlet P, Stewart D, Gielly L, Hausser J, Vogel P (1999) Molecular phylogeny and evolution of Sorex shrews (Soricidae: Insectivora) inferred from mitochondrial DNA sequence data. Mol Phylogenet Evol 11: 222-235.

Ibrahim SF, van den Engh, G (2004) High-speed chromosome sorting. Chromosom Res 12: 5-14.

Lugon-Moulin N, Brünner H, Wyttenbach A, Hausser J, Goudet J (1999) Hierarchical analyses of genetic differentiation in a hybrid zone of Sorex araneus (Insectivora: Soricidae). Mol Ecol 8: 419-431.

Lugon-Moulin N, Balloux F, Hausser J (2000) Genetic differentiation of common shrew (Sorex araneus) populations among different alpine valleys revealed by microsatellites. Acta Theriol 45: 103-117.

Murphy WJ, Pevzner PA, O'Brien, SJ (2004) Mammalian phylogenomics comes of age. Trends Genet 20: 631-639.

Navarro A, Barton NH (2003) Accumulating postzygotic isolation genes in parapatry: a new twist on chromosomal speciation. Evolution 57: 447-459.

Noor MAF, Grams KL, Bertucci LA, Reiland J (2001) Chromosomal inversions and the reproductive isolation of species. Proc Natl Acad Sci USA 98: 12084-12088.

Nowak RM (1991) Walker's Mammals of the World, 5th edn. Baltimore, MD: Johns Hopkins University Press.

O'Brien SJ, Menotti-Raymond M, Murphy WJ et al. (1999) The promise of comparative genomics in mammals. Science $\mathbf{2 8 6}$ : 458-481.

O'Brien SJ, Eizirik E, Murphy WJ (2001) On choosing Mammalian genomes for sequencing. Science 292: 2264-2266.

Pack SD, Borodin PM, Serov OL, Searle JB (1993) The Xautosome translocation in the common shrew (Sorex araneus L.): late replication in female somatic cells and pairing in male meiosis. Chromosoma 102: 355-360.

Pack SD, Kolonin MG, Borodin PM, Searle JB, Serov OL (1995) Gene mapping in the common shrew (Sorex araneus; Insectivora) by shrew-rodent cell hybrids: chromosome localization of the loci for HPRT, TK, LDHA, MDH1, G6PD, PGD, and ADA. Mamm Genome 6: 784-787.

Panithanarak T, Hauffe HC, Dallas JF, Glover A, Ward RG, Searle JB (2004) Linkage-dependent gene flow in a house mouse chromosomal hybrid zone. Evolution 58: 184-192.

Pennisi E (2004) More genomes, but shallower coverage. Science 304: 1227.
Rieseberg LH (2001) Chromosomal rearrangements and speciation. Trends Ecol Evol 16: 351-358.

Rieseberg LH, Whitton J, Gardner K (1999) Hybrid zones and the genetic architecture of a barrier to gene flow between two sunflowers species. Genetics 152: 713-727.

Samonte RV, Eichler EE (2002) Segmental duplication and the evolution of the primate genome. Nat Rev Genet 3: 65-72.

Sargan DR, Yang F, Squire M, Milne BS, O'Brien PCM, Ferguson-Smith MA (2000) Use of flow-sorted canine chromosomes in the assignment of canine linkage, radiation hybrid, and syntenic groups to chromosomes: refinement and verification of the comparative chromosome map for dog and human. Genomics 69: 182-195.

Searle JB, Wójcik, JM (1998) Chromosomal evolution: the case of Sorex araneus. In Wójcik JM, Wolsan M, eds. Evolution of Shrews. Bialowieza: Mammal Research Institute, Polish Academy of Sciences, pp. 219-262.

Searle JB, Fedyk S, Fredga K, Hausser J, Volobouev VT (1991) Nomenclature for the chromosomes of the common shrew Sorex araneus. Mém Soc Vaud Sci Nat 19: 13-22.

Sharman GB (1956) Chromosomes of the common shrew. Nature 177: $941-942$.

Telenius H, Pelmear AH, Tunnacliffe A et al. (1992) Cytogenetic analysis by chromosome painting using DOP PCR amplified flow sorted chromosomes. Gene Chromosomes Cancer 4: 257-263.

Thomas EE, Srebro N, Sebat J et al. (2004) Distribution of short paired duplications in mammalian genomes. Proc Natl Acad Sci USA 101: 10349-10354.

Volobouev VT (1989) Phylogenetic relationships of the Sorex araneus-arcticus species complex (Insectivora, Soricidae) based on high-resolution chromosome analysis. J Heredity 80: 284-290.

Volobouev VT, Catzeflis, F (1989) Mechanisms of chromosomal evolution in three European species of the Sorex araneusarcticus group (Insectivora: Soricidae). Z zool Syst Evolut-forsch 27: 252-262.

Wójcik JM, Searle JB (1988) The chromosome complement of Sorex granarius-the ancestral karyotype of the common shrew (Sorex araneus)? Heredity 61: 225-229.

Wójcik JM, Ratkiewicz M, Searle, JB (2002) Evolution of the common shrew Sorex araneus: chromosomal and molecular aspects. Acta Theriol 47: 139-167.

Wójcik JM, Borodin PM, Fedyk S, et al. (2003) The list of the chromosome races of the common shrew Sorex araneus (updated 2002). Mammalia 67: 169-178.

Wyttenbach A, Favre L, Hausser J (1997) Isolation and characterization of simple sequence repeats in the genome of the common shrew. Mol Ecol 6: 797-800.

Yang F, Carter NP, Shi L, Ferguson-Smith MA (1995) A comparative study of karyotypes of muntjacs by chromosome painting. Chromosoma 103: 642-652.

Yang F, O'Brien PCM, Milne BS et al. (1999) A complete comparative chromosome map for the dog, red fox, and human and its integration with canine genetic maps. Genomics 62: 189-202.

Zhdanova NS, Fokina VM, Balloux F et al. (2003) Current cytogenetic map of the common shrew, Sorex araneus L.: localization of 7 genes and 4 microsatellites. Mammalia 68: 285-293. 